\title{
Isolation and characterization of stem cell-like precursor cells from primary human anaplastic oligoastrocytoma
}

\author{
Liang $\mathrm{Yi}^{1, *}$, Zhi-hua Zhou ${ }^{1, *}$, Yi-fang Ping ${ }^{1, *}$, Jian-hong Chen ${ }^{1}$, Xiao-hong Yao ${ }^{1}$, Hua Feng ${ }^{2}$, \\ Jia-you Lu ${ }^{2}$, Ji Ming Wang ${ }^{3}$ and Xiu-wu Bian ${ }^{1}$ \\ ${ }^{1}$ Institute of Pathology, Southwest Hospital, Third Military Medical University, Chongqing, China; \\ ${ }^{2}$ Department of Neurosurgery, Southwest Hospital, Third Military Medical University, Chongqing, China and \\ ${ }^{3}$ Laboratory of Molecular Immunoregulation, Cancer and Inflammation Program, Center for Cancer Research, \\ National Cancer Institute at Frederick, Frederick, MD, USA
}

\begin{abstract}
A small population of stem cell-like precursors in solid tumors are linked to histological composition, progression, angiogenesis, metastasis, recurrence and drug resistance of a variety of malignant tumors. Oligoastrocytoma is the most common brain mixed glioma composed of mixed cells of oligodendroglial and astrocytic phenotypes. Identification and characterization of stem cell-like precursors in oligoastrocytoma may shed light on the oncogenesis of this unique type of tumor and assist in the design of novel therapeutic strategy. Here, tumor stem cell-like precursors were identified from primary human anaplastic oligoastrocytomas by labeling of the tumor sections with nestin and CD133. Tumor cells were cultured in vitro in stem cell medium with growth factors and the capacity of the surviving stem cell-like precursors to form tumor spheres was tested. The tumor spheres were further injected subcutaneously into nude mice to observe the contribution of stem cell-like precursors to histological composition and tumor progression. We found that primary human oligoastrocytoma tissues contained nestin ${ }^{+} / \mathrm{CD} 133^{+}$stem cell-like precursors. These cells differentiated into tumor cells with both oligodendroglial and astrocytic characteristics and formed tumor spheres in vitro, which upon implantation in nude mice, grew into tumor nodules containing nestin ${ }^{+} / \mathrm{CD} 133^{+}$cells at levels higher than in the primary tumor tissues. This study revealed for the first time that anaplastic human oligoastrocytomas contained stem cell-like precursors, which exhibit neural stem cell properties with tumorigenicity. These stem cell-like precursors may be responsible for the oligodendroglial and astrocytic components of human oligoastrocytoma and should be considered as therapeutic targets.

Modern Pathology (2007) 20, 1061-1068; doi:10.1038/modpathol.3800942; published online 27 July 2007
\end{abstract}

Keywords: oligoastrocytoma; cancer stem cell; CD133; nestin; differentiation

The theory of tumor stem cells (TSCs), or cancer stem cells, implies that, similar to normal tissues, malignant tumors might also 'develop' from somatic stem cells that accumulated mutations leading to transformation. ${ }^{1}$ Although this theory met skepticism, over the past decade there has been abundant evidence to support the role of embryonic and fetal stem cells in oncogenesis. ${ }^{2}$ It has been found that mutations occurring during expansion phases of totipotential stem cells throughout development

Correspondence: Dr X-W Bian, PhD, Institute of Pathology, Southwest Hospital, Third Military Medical University, Gaotanyan, Chongqing 400038, China.

E-mail: bianxiuwu@263.net

* These authors contributed equally to this work.

Received 14 May 2007; revised 24 June 2007; accepted 25 June 2007; published online 27 July 2007 may prompt the formation of tumors in adulthood. In mature adult tissues, stem cells and progenitor cells may also constitute the sources of oncogenic transformation following somatic mutations, as shown by the isolation of TSCs from human acute myeloid leukemia (AML) ${ }^{3-6}$ and breast cancer. ${ }^{7-9}$

In tumors of the central nervous system (CNS), TSCs have also been isolated and successfully maintained in culture from medulloblastoma, ${ }^{10}$ glioblastoma, ${ }^{11-18}$ and ependymoma, ${ }^{19}$ as well as from several glioma cell lines. ${ }^{17,20}$ These results suggest that brain tumors may contain a subpopulation of precursor cells that share similar surface markers and properties with neural stem cells. Oligoastrocytoma is a CNS tumor type composed of a mixture of cells of astrocytic and oligodendrocytic origin. Since this tumor type is second only to astrocytomas in the rate of incidence among CNS 
tumors $^{21}$ and is often lethal, it is important to investigate the existence of TSCs so as to gain insight into tumor development, progression and the cause for mixed histological pattern. In this study, we report the isolation of tumor-stem cell like precursors from human primary oligoastrocytoma tissues and characterization of the contribution of such precursor cells to the unique tumor histology and progression.

\section{Materials and methods}

\section{Culture of Tumor Spheres from Primary Human Oligoastrocytoma Tissues}

Tumor tissues from three cases of anaplastic oligoastrocytoma were obtained with the informed consent of the patients as approved by the institutional Research Ethics Board at Southwest Hospital, Chongqing, China, PR. Tumor tissues were used for primary cell culture and immunohistochemical staining. For primary tumor cell culture, the tissues were cut into $1 \times 1 \times 1 \mathrm{~mm}^{3}$ pieces and cultured in $50-\mathrm{mm}^{2}$ flasks in $0.5 \mathrm{ml}$ fetal calf serum (FCS). After $4 \mathrm{~h}$, the flasks were replenished with $3 \mathrm{ml}$ DMEM (Gibco, USA) containing 10\% FCS. When a monolayer of primary tumor cells was formed, the cells were detached with $0.25 \%$ trypsin (Gibco) and suspended in defined stem cell medium, that is, DMEM/F12 (Gibco) containing $20 \mathrm{ng} / \mathrm{ml}$ basic fibroblast growth factor (bFGF, Peprotech, USA), $20 \mathrm{ng} /$ ml epidermal growth factor (EGF, Peprotech), B27 Supplement ( $1 \times$, Gibco), $2 \mathrm{mmol} / \mathrm{l}$ L-glutamine and $4 \mathrm{U} / \mathrm{l}$ insulin. The culture media were replaced every 4 days. When the primary neurospheres were passed, we dissociated and plated the cells from the neurospheres in 96-well plates at a density of 10 cells/well in $0.2 \mathrm{ml}$ of the defined stem cell medium to acquire the secondary neurospheres.

\section{Immunocytochemistry of Tumor Spheres}

The spheres formed by primary tumor cells were placed onto coverslips precoated with poly-L-lysine (Sigma, USA) in DMEM containing 10\% FCS. The spheres were fixed with $4 \%$ paraformaldehyde for $20 \mathrm{~min}$ at room temperature, treated with normal goat serum (Boshide Biotech, China) and then were incubated with the following antibodies: rabbit antihuman nestin polyclonal antibody (1:100 dilution, Chemicon, USA) and mouse anti-human CD133/1 monoclonal antibody (1:50 dilution, Milteny Biotech, Germany). The primary antibodies bound on tumor cells were reacted with FITC-conjugated goat anti-rabbit or anti-mouse IgG (1:50, Sigma). The cells were then counterstained with 4',6-diamidino2-phenylindole (DAPI, $100 \mu \mathrm{g} / \mathrm{ml}$, Sigma) or propidium iodide (PI, $5 \mu \mathrm{g} / \mathrm{ml}$, Sigma) to reveal the nuclei.

For testing the differentiation potential of the neurospheres formed by the TSCs, the secondary tumor spheres were seeded onto coverslips in DMEM containing 10\% FCS. The differentiation markers including glial fibrillary acidic protein (GFAP) for astrocytes and myelin basic protein (MBP) for oligodendrocytes as well as CD133 for stem cells were detected by immunocytochemistry on the coverslips with the spheres. Briefly, the cells were fixed with $4 \%$ paraformaldehyde for $20 \mathrm{~min}$ at room temperature, treated with normal goat serum and then stained with the following antibodies: rabbit anti-human GFAP polyclonal antibody (1:100 dilution, Zhongshan Jinqiao Biotech, China), rabbit anti-human MBP polyclonal antibody (1:200 dilution, Boshide Biotech, China). The primary antibodies were bound by TRITC-conjugated goat anti-rabbit IgG (1:100, Sigma) and FITC-conjugated goat anti-rabbit IgG (1:50, Sigma) respectively. The cells were counterstained with DAPI to reveal the nuclei.

\section{Implantation of Tumor Spheres in Nude Mice}

To evaluate the in vivo tumorigenicity of CD133 ${ }^{+}$ cells from the neurospheres, 4 to 6-week-old athymic nu/nu mice (BALB/c, Laboratory Animal Center, Third Military Medical University) were subcutaneously implanted with isolated CD133+ sphere cells and $\mathrm{GFAP}^{+} / \mathrm{CD} 133^{-}$differentiated tumor cells respectively $\left(5 \times 10^{3}\right.$ and $5 \times 10^{4}$ per mouse, five mice for each group) in $100 \mu \mathrm{l}$ of serumfree DMEM/F12 in the right chest. Tumor volume $\left(\mathrm{T}_{\mathrm{V}}\right)$ was calculated by using the following formula: $\mathrm{T}_{\mathrm{V}}($ tumor volume $)=l$ (length) $\times w^{2}$ (width) $/ 2$. At the tenth week after implantation, the mice were killed and tumors were removed for histological examination and immunohistochemistry for CD133, nestin, GFAP and MBP. Mice were hosted according to the guidelines of the institutional Committee for Laboratory Animal Usages.

\section{Immunohistochemical Staining of Tumor Tissues}

For examination of TSCs in tumor tissues, nestin and CD133 were detected with immunofluorescence on cryostat sections. The specimens from either human specimens or nude mice were fixed in $4 \%$ paraformaldehyde, embedded in Tissue-Tek OCT (optimal cutting temperature) compound and frozen at $-20^{\circ} \mathrm{C}$ for cryostat sections $(8 \mu \mathrm{m})$, which were mounted on poly-L-lysine-coated slides, and airdried for $24 \mathrm{~h}$. The sections were pretreated with normal goat serum for $30 \mathrm{~min}$ at $37^{\circ} \mathrm{C}$ and then incubated with primary antibody (rabbit anti-human nestin or mouse anti-human CD133 antibody) overnight, followed by incubation with FITC- or TRITCconjugated secondary antibody (goat anti-rabbit or anti-mouse IgG). For double labeling of nestin and CD133 on the sections, both the primary antibodies were used overnight, and both TRITC-conjugated goat anti-rabbit IgG and FITC-conjugated IgG were 
used as secondary antibodies. The sections were counterstained with DAPI to reveal the nuclei. The stained sections were examined and photographed with a laser confocal scanning microscope (Leica, Germany).

To detect GFAP and MBP, two differentiation markers for the tumor cells, in the tumor tissues, paraffin-embedded sections were used for immunohistochemistry. The tumor tissues were fixed in 10\% phosphate-buffered formalin and embedded in paraffin for sectioning. The tumor sections were incubated with rabbit anti-human GFAP or antihuman MBP polyclonal antibody, followed by detection using the ChemMate Detection kit (Dako). A positive reaction was indicated by brown color using $\mathrm{DAB}$, and was counterstained with hematoxylin.

Cells positive for GFAP, MBP or CD133 were quantified by counting all stained cells within 20 randomly selected microscopic fields, and the percentages were calculated on the basis of the total number of nuclei counted.

\section{Statistical Analysis}

Parametric data were expressed as the mean values \pm standard deviation (s.d.). Data were analyzed with computer aided SPSS10.0 statistical software. When two groups were compared, the unpaired dependent-samples $t$-test was used. A value of $P<0.05$ was considered statistically significant.

\section{Results}

\section{Histopathology and Immunohistochemistry of Primary Human Tumor Tissues}

All three cases of anaplastic oligoastrocytoma were located in the cerebral hemispheres, compressing the ventricles (Figure 1a-c). The tumor tissues used for primary culture had typical histologic features of anaplastic oligoastrocytomas. Immunostaining showed various numbers of GFAP-positive tumor cells mixed with MBP-positive tumor cells, representing two types of distinctly differentiated tumor cells (Figure 1d-f). To identify the stem cell-like precursors, or TSCs, we used either single or double immunofluorescence staining with antibodies to nestin and CD133. We found a few tumor cells positive for nestin in the cytoplasm or CD133 on the cell membrane (Figure 2a-j).

\section{Formation of Tumor Spheres}

Dissociated primary tumor cells were seeded for $24 \mathrm{~h}$ in serum-free medium supplemented with EGF and bFGF. Most of the tumor cells became adherent, with a minority of floating cells forming spheres composed of two to three cells. After an additional $48 \mathrm{~h}$, the floating spheres were expanded to contain 10-20 tumor cells, with bright appearance and a sharp edge. On the sixth day, the spheres further expanded, with cell numbers in each sphere ranging from 100 to 200 (Figure 3a-c). The center of the
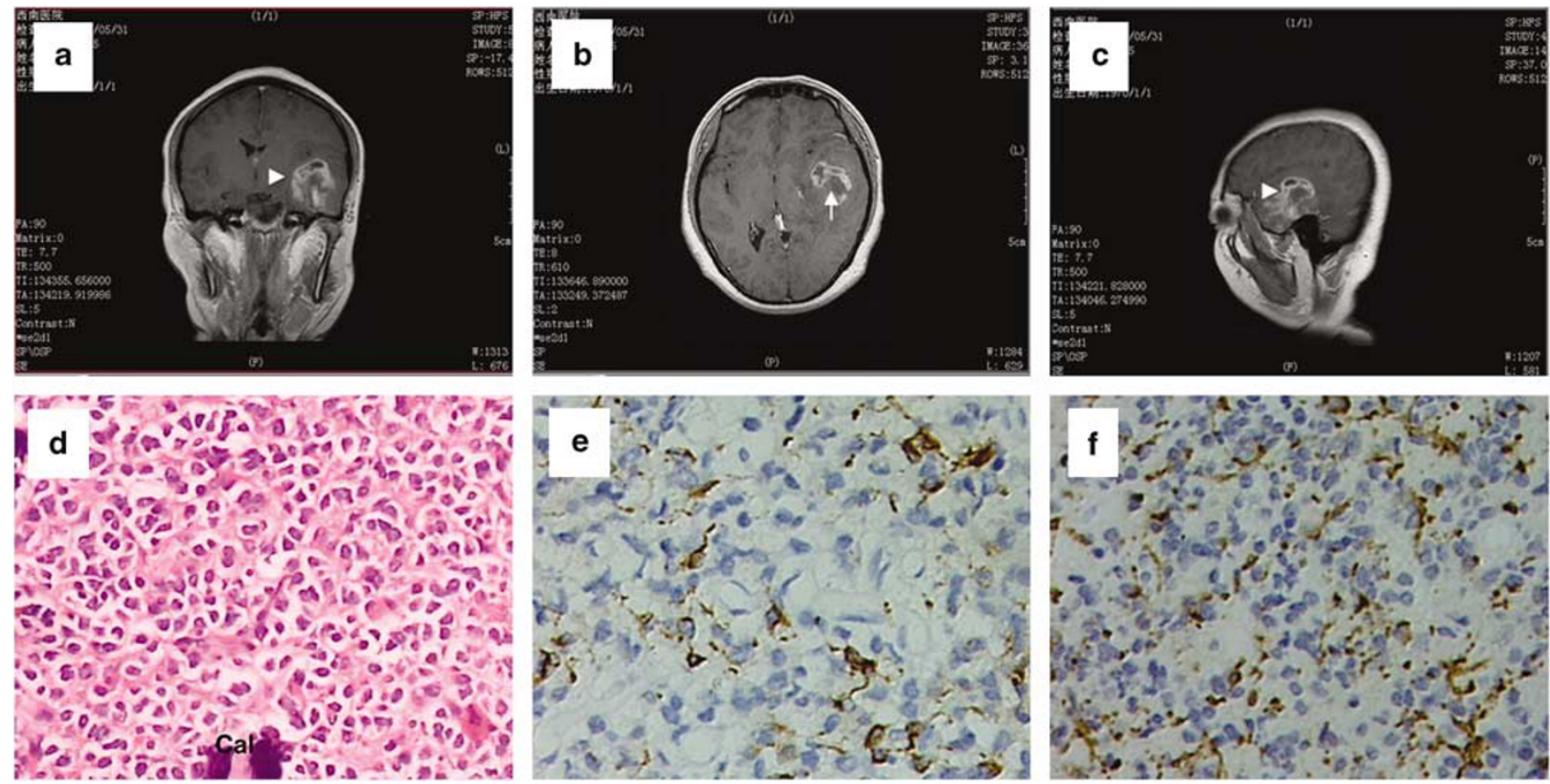

Figure 1 Magnetic resonance images, histopathology and immunohistochemistry of a case of cerebral oligoastrocytoma. (a-c) Magnetic resonance images showing a tumor (white arrowheads) located in the left temporal lobe. With contrast medium an irregular low-signal area (white arrow) in the tumor is visible. (d) Routine section shows a typical anaplastic oligoastrocytoma with calcification (Cal). Immunohistochemistry showed that a few tumor cells are positive for GFAP (e), while others are positive for MBP (f). (d), HE; (e and f) positive brown color was revealed by DAB and counterstained with hematoxylin. 

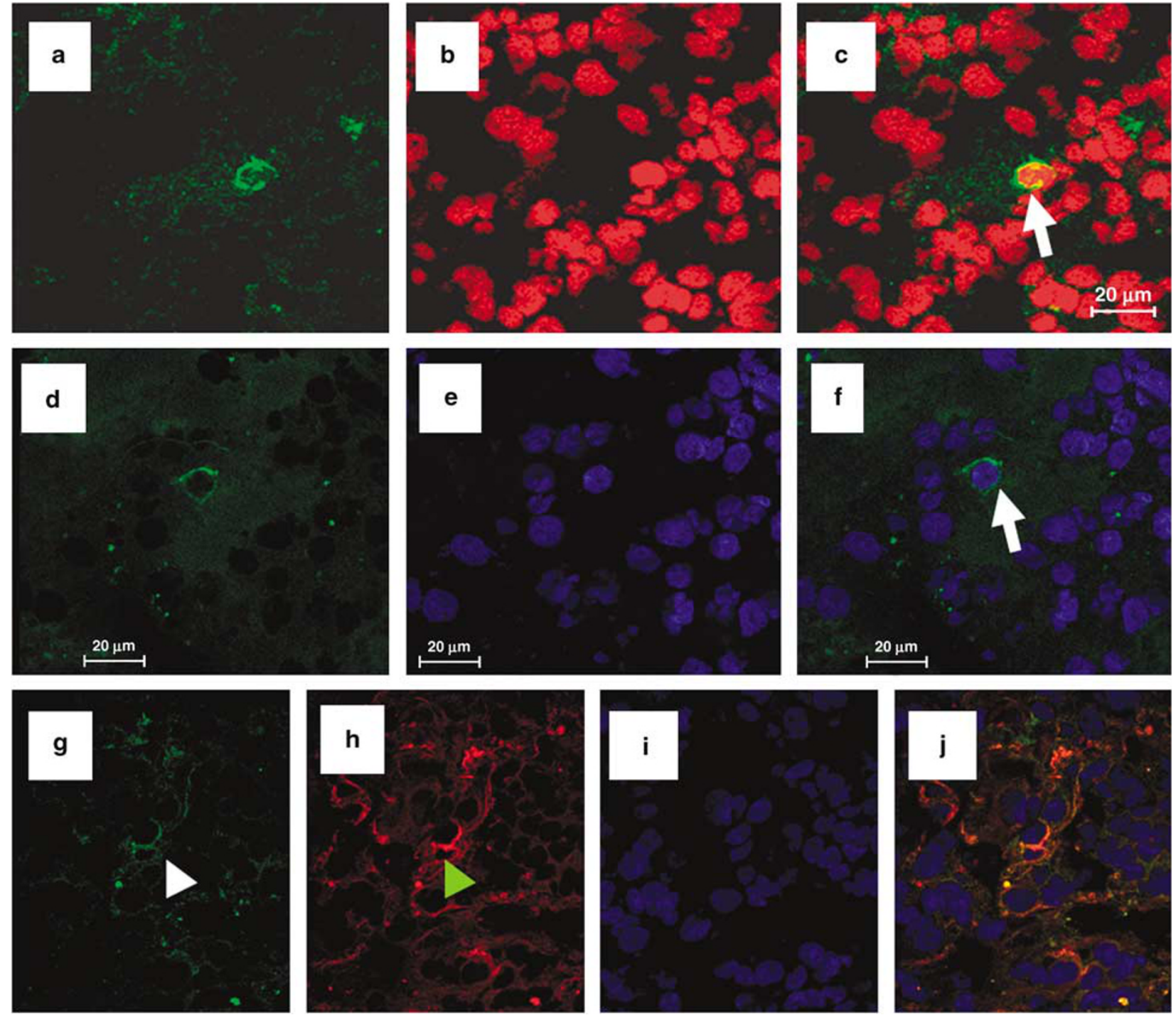

Figure 2 Stem cell-like tumor cells detected in primary tumor samples. Tissue-Tek OCT (optimal cutting temperature) compoundembedded sections were labeled with antibodies to CD133 (a-c, green, PI-counterstained nuclei in red) or nestin (d-f, green) to identify stem cell-like tumor cells (white arrows). Areas indicated by arrowheads in (g) and (h) represent CD133 (green) and nestin (red) double staining. DAPI-counterstained nuclei are in blue (e, i). (c, f and $\mathbf{j})$ Merged images. Laser confocal scanning microscopy. Scale bars $=20 \mu \mathrm{m}$.

spheres became dark and passages were made at this time point.

\section{Phenotype of Tumor Spheres}

We stained tumor cells in the spheres with immunofluorescence of neural stem cell markers CD133 and nestin. Tumor cells in the spheres expressed both CD133 and nestin (Figure 3d-i), implying stem cell phenotype, that is, stem cell-like glioma cells or glioma stem cells. For analysis of cell differentiation, tumor spheres were cultured in DMEM containing $10 \%$ FCS. After $8 \mathrm{~h}$, all spheres adhered steadily to the plastic flasks, without tumor cells migrating from the spheres, whereas a small proportion of the tumor cells migrated out from the spheres after $24 \mathrm{~h}$ in culture. On the third day, more tumor cells migrated to greater distances and the tumor spheres flattened. On day 7 , proliferating tumor cells covered the bottoms of the flasks and masked primary tumor cells. The proliferating tumor cells expressed a moderate level of GFAP and MBP as compared with primary cells (Figure 4). These results indicate that sphere forming primary tumor cells are progeny cells capable of proliferation and differentiation.

\section{Tumorigenicity of Tumor Spheres}

Tumor spheres were transplanted s.c. to nude mice and tumor nodules were palpable after 9 weeks in mice implanted with $5 \times 10^{4} \mathrm{CD}_{133^{+}} /$nestin $^{+}$cells. 

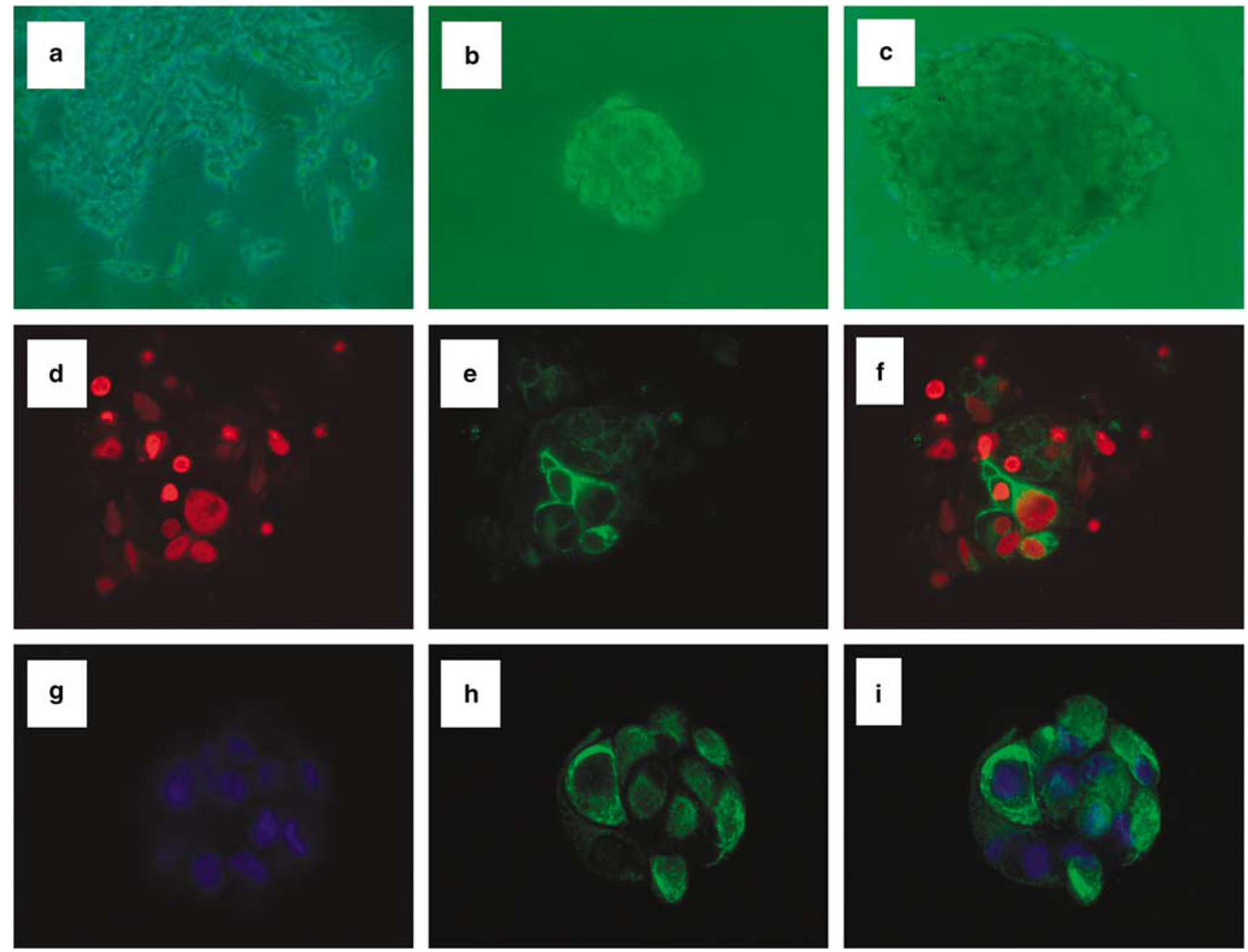

Figure 3 Tumor cells in primary culture and clones formed by isolated stem cell-like precursors (SCPs). (a) Tumor cells creeping out from the edges of tissue pieces at $96 \mathrm{~h}$ after plating in DMEM containing 10\% FCS. (b and c) Neurosphere-like colonies formed by SCPs on day 3 (b) and day 6 (c) in stem cell medium. (d-i) Immunofluorescence of stem cell makers CD133 (d-f, counterstained with PI showing nuclei in red) and nestin (g-i, counterstained with DAPI showing nuclei in blue). (a-c) Phase contrast microscopy. (d-i) Laser confocal scanning microscopy. (f, i) Merged images.

The average volume of the tumors reached $0.64 \pm 0.02 \mathrm{~cm}^{3}$ at the tenth week after implantation, significantly larger compared with $0.38 \pm 0.08 \mathrm{~cm}^{3}$ in mice implanted with $5 \times 10^{3} \mathrm{CD} 133^{+} /$nestin $^{+}$cells $(P<0.05)$. Immunostaining showed various numbers of GFAP-positive tumor cells mixed with MBP positive tumor cells (Figure 5). Tumor tissue sections were found to contain dispersed CD133and nestin-positive tumor cells, with the positive rate significantly higher than in the primary tumors (Figure 6). No tumors were formed in mice implanted with $\mathrm{GFAP}^{+} / \mathrm{CD} 133^{-}$-differentiated cells. These results suggest that $\mathrm{CD}_{133^{+}}$stem cell-like precursors derived from anaplastic oligoastrocytoms are tumorigenic at low cell numbers.

\section{Discussion}

In the 1970s, cancer cells derived from a single tumor showed different ability to form clones in vitro. This has led to the vigorous studies of the presence of stem-like cells that may constitute the origin of malignant tumors. In fact, 'tumor stem cells' have been isolated from a variety of malignant human solid tumors. As compared with committed tumor cells, stem-like tumor cells not only possess the property of self-renewal and multipotent differentiation, but also are more pro-angiogenic and resistant to radio- or drug therapy. ${ }^{22-24}$ Tumor stem cells are considered as the origin of the initiation, progression, metastasis and recurrence of malignant tumors. ${ }^{25-27}$ Thus far, brain tumor stem cells have been isolated from malignant fetal neuroepithelial tumors or glioblastoma multiforme. Our study further provides evidence for the existence of stem cell-like precursors in human oligoastrocytoma, with the expression of nestin and CD133, two key markers of neural stem cells.

We found that tumor spheres formed by cells from oligoastrocytomas expressed CD133 and nestin, and 

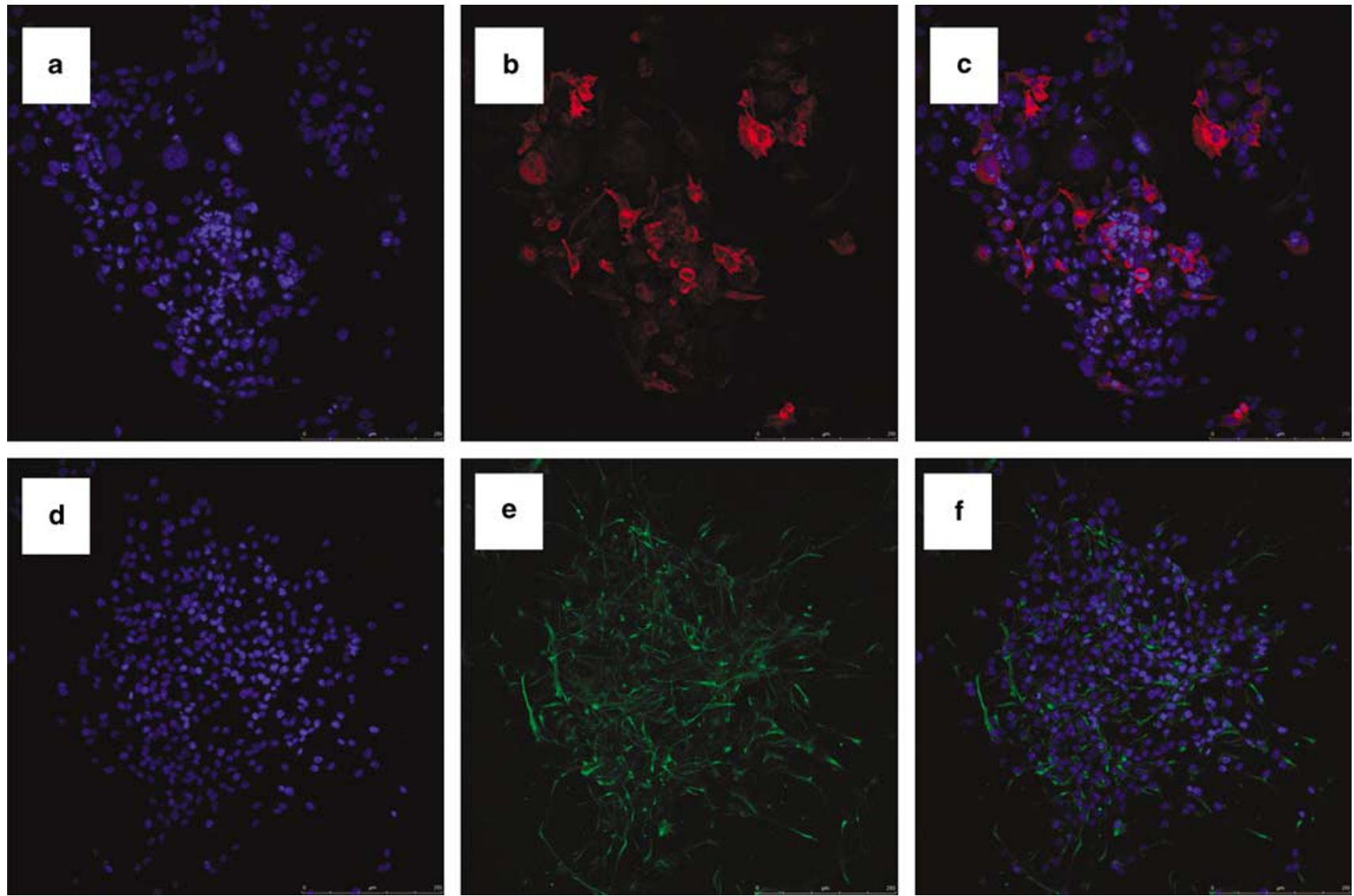

Figure 4 Differentiating cells from neurospheres. Cells fixed in 4\% paraformaldehyde were labeled with anti-GFAP antibody to reveal positive cells (a-c, red). Antibody to MBP was used to detect MBP-positive cells (d-f, green). (c) and (f) are merged images. (a-f) DAPIcounterstained nuclei in blue. Laser confocal scanning microscopy.
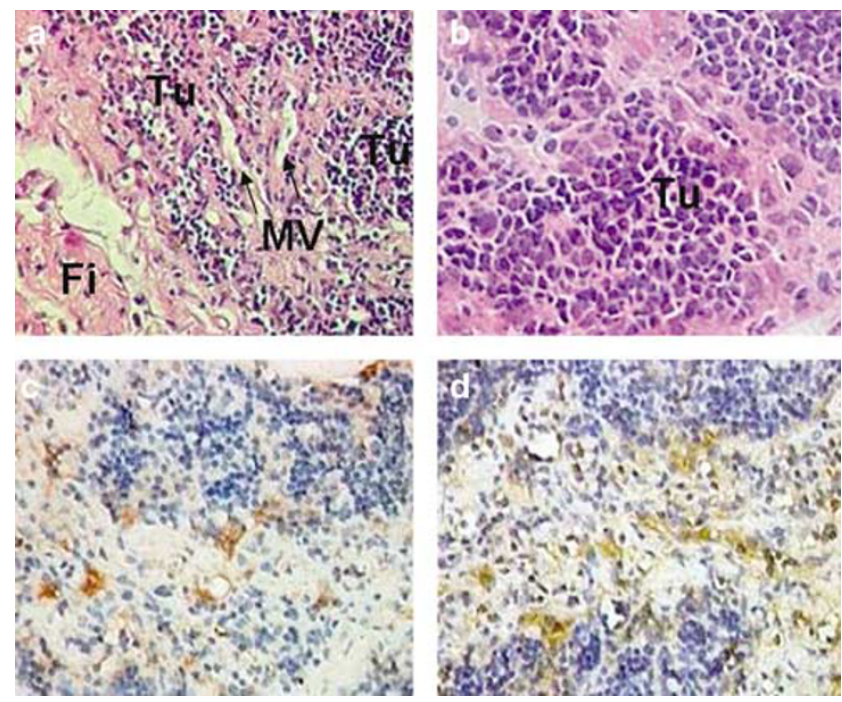

Figure 5 Morphology and immuohistochemistry of subcutaneous tumor xenografts. (a-b) $\mathrm{CD}_{133^{+}}$cells form nodular tumors (Tu) with numerous microvessels (MV), and tumor cells infiltrate the surrounding fibrous tissues (Fi). (c, d) At the edge of the tumor, well-differentiated tumor cells are positive for GFAP (c) and BMP (d), indicating the tendency of astrocytic and oligodendrocytic differentiation. (a and b), HE; (c and d), immunohistochemistry with positive reaction in brown color revealed by DAB and counterstained with hematoxylin. the cells derived from these tumor spheres possessed the properties of stem cells. However, these CD133- ${ }^{-}$cells may not represent bona fide glioblastoma stem cells. We therefore further studied the function of these CD133 ${ }^{+}$cells. Secondary spheres were acquired from diluted cloning, indicating that these cells possessed the property of self-renewal. $\mathrm{CD}_{133^{+}}$sphere cells negative for either GFAP or MBP could differentiate into adhesive cells positive for GFAP or MBP, suggesting their property of multilineage differentiation. Additionally, the sphere cells were tumorigenic when implanted in nude mice, therefore, $\mathrm{CD} 133^{+}$cells isolated from human oligoastrocytoma cells exhibited characteristics of stem cells.

As a common malignancy in the brain, oligoastrocytoma is positive both for the oligodendroglial marker MBP and the astrocytic marker GFAP. In our study, the primary human tumors were diagnosed as anaplastic oligoastrocytomas (WHO grade III), which were both MBP and GFAP positive. The tumors contained dispersed nestin- and CD133positive cells, which have been thought to be the origin of oligoastroglioma. ${ }^{21}$ Based on the 'double lineage' theory, committed oligodendrocytes and type II astrocytes are progeny derived from 

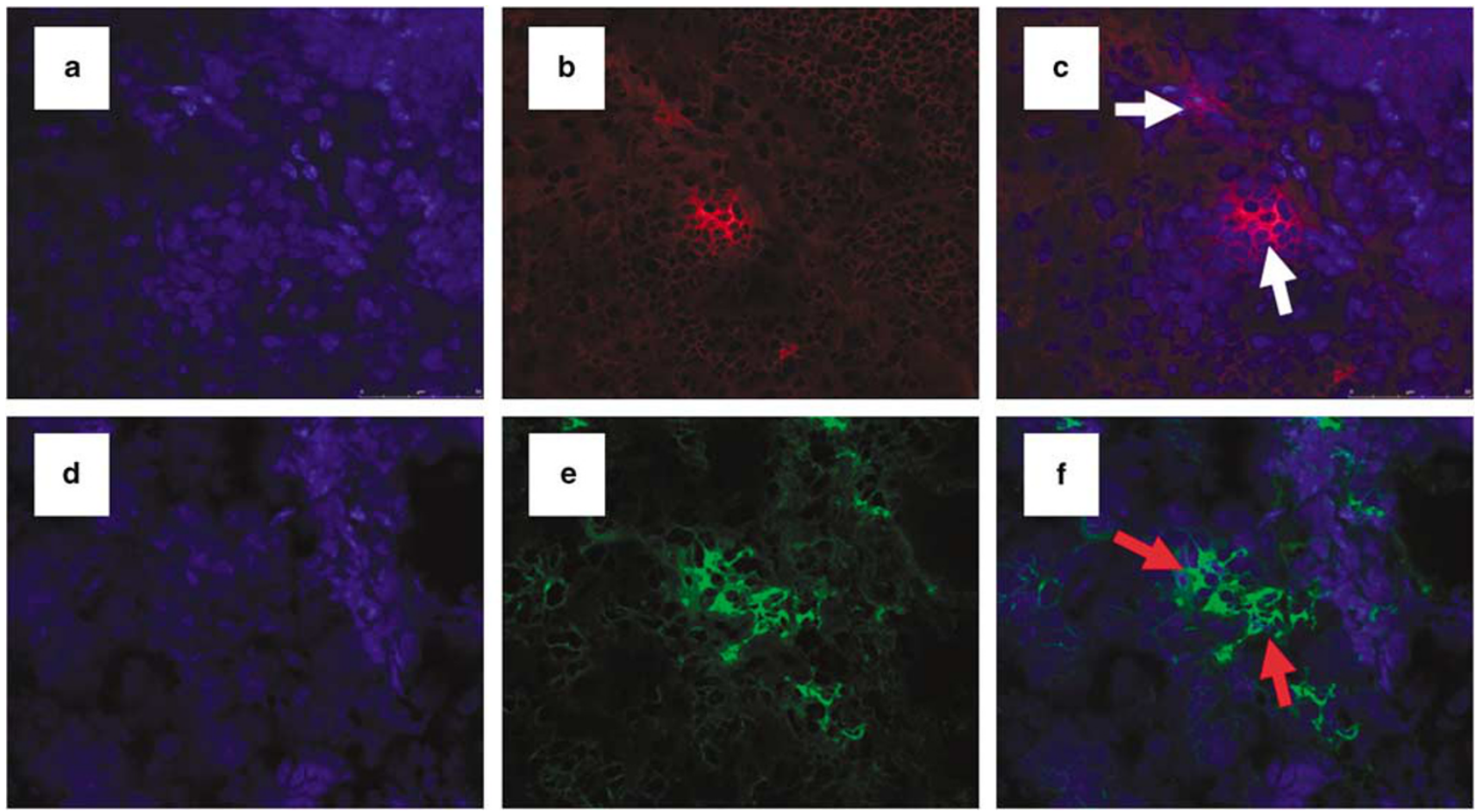

Figure 6 Stem cell-like tumor cells (STCs) detected in frozen xenograft tumor tissues. (a-c) STCs were labeled with anti-CD133 (red, white arrows) or anti-nestin antibody (d-f, green, red arrows), with nuclei counterstained by DAPI shown in blue. Higher number of STCs is seen in the xenograft tumor tissues than in the primary tumors. (c, f) Merged images. Laser confocal scanning microscopy.

oligodendrocyte type II astrocyte $\left(\mathrm{O}_{2} \mathrm{~A}\right)$ progenitors. As the result of transformation of $\mathrm{O}_{2} \mathrm{~A}$ progenitors or their parental generation, it appears reasonable to explain why the rate of oligoastrocytomas is rather frequent in mixed gliomas, consistent with the interpretation of the origin of tumors by 'cancer stem cell' hypothesis.

Self-renewal and differentiation are basic properties of stem cells. ${ }^{28}$ When cultured in serum-free medium, committed tumor cells usually cease propagation, whereas tumor stem cells continue to proliferate. We found that tumor cells in the spheres formed by primary oligoastrocytoma stem cell-like precursors expressed both nestin and CD133. When recultured in medium containing FCS, tumor spheres became adherent and their progeny cells resembled the primary tumor cells. In order to distinguish stem cell-like precursors isolated from oligoastrocytoma from normal neural stem cells, we implanted tumor spheres formed by stem cell-like precursors into nude mice. The xenograft tumors showed a latent time much longer than the conventional xenografts, ${ }^{29}$ implying the tumorigenicity of these stem cell-like precursors and their potential to act as the source of tumor recurrence during therapy. Thus, our successful isolation and characterization of stem-like cells will provide further insight into the understanding of the development and progression of human anaplastic oligoastrocytoma and assist in the design of more effective therapeutic means for this lethal tumor in the brain.

\section{Acknowledgements}

We thank technicians Wei Sun and Li-ting Wang (Central Laboratory, Third Military Medical University, Chongqing, China) for their technical assistance in laser confocal scanning microscopy. This project was supported by grants from National Natural Science Foundation of China (NSFC, no. 30670804), the National Basic Research Program (973 Program, No. 2006CB708503) and National High-tech R\&D Program (863 Program, no. 2006AA02Z475).

\section{References}

1 Reya T, Morrison SJ, Clarke MF, et al. Stem cells, cancer, and cancer stem cells. Nature 2001;414: 105-111.

2 Frank SA, Nowak MA. Cell biology: developmental predisposition to cancer. Nature 2003;422:494.

3 Bonnet D, Dick JE. Human acute myeloid leukemia is organized as a hierarchy that originates from a primitive hematopoietic cell. Nat Med 1997;3:730-737.

4 Passegue E, Jamieson CM, Ailles LE, et al. Normal and leukemic hematopoiesis: are leukemias a stem cell disorder or a reacquisition of stem cell characteristics? Proc Natl Acad Sci USA 2003;100:11842-11849.

5 Guan YH, Gerhard B, Hogge DE. Detection, isolation, and stimulation of quiescent primitive leukemic progenitor cells from patients with acute myeloid leukemia (AML). Blood 2003;101:3142-3149. 
6 Hope KJ, Jin L, Dick JE. Acute myeloid leukemia originates from a hierarchy of leukemic stem cell classes that differ in self-renewal capacity. Nat Immunol 2004; 5:738-743.

7 Al-Hajj M, Wicha MS, Benito-Hernandez A, et al. Prospective identification of tumorigenic breast cancer cells. Proc Natl Acad Sci USA 2003;100:3983-3988.

8 Ponti D, Costa A, Zaffaroni $\mathrm{N}$, et al. Isolation and in vitro propagation of tumorigenic breast cancer cells with stem/progenitor cell properties. Cancer Res 2005; 65:5506-5511.

9 Youn BS, Sen A, Behie LA. Scale-up of breast cancer stem cell aggregate cultures to suspension bioreactors. Biotechnol Prog 2006;22:801-810.

10 Hemmati HD, Nakano I, Lazareff JA, et al. Cancerous stem cells can arise from pediatric brain tumors. Proc Natl Acad Sci USA 2003;100:15178-15183.

11 Tunici P, Bissola L, Lualdi E, et al. Genetic alterations and in vivo tumorigenicity of neurospheres derived from an adult glioblastoma. Mol Cancer 2004;3:25.

12 Ignatova TN, Kukekov VG, Laywell ED, et al. Human cortical glial tumors contain neural stem-like cells expressing astroglial and neuronal markers in vitro. Glia 2002;39:193-206.

13 Singh SK, Clarke ID, Terasaki M, et al. Identification of a cancer stem cell in human brain tumors. Cancer Res 2003;63:5821-5828.

14 Galli R, Binda E, Orfanelli U, et al. Isolation and characterization of tumorigenic, stem-like neural precursors from human glioblastoma. Cancer Res 2004;64: 7011-7021.

15 Singh SK, Hawkins C, Clarke ID, et al. Identification of human brain tumour initiating cells. Nature 2004; 432:396-401.

16 Yuan XP, Curtin J, Xiong YZ, et al. Isolation of cancer stem cells from adult glioblastoma multiforme. Oncogene 2004;23:9392-9400.

17 Kang SK, Park JB, Cha SH. Multipotent, dedifferentiated cancer stem-like cells from brain gliomas. Stem Cells Dev 2006;15:423-435.
18 Lee JW, Kotliarova S, Kotliarov Y, et al. Tumor stem cells derived from glioblastomas cultured in bFGF and EGF more closely mirror the phenotype and genotype of primary tumors than do serum-cultured cell lines. Cancer Cell 2006;9:391-403.

19 Taylor MD, Poppleton H, Fuller C, et al. Radial glia cells are candidate stem cells of ependymoma. Cancer Cell 2005;8:323-335.

20 Kondo T, Setoguchi T, Taga T. Persistence of a small subpopulation of cancer stem-like cells in the C6 glioma cell line. Proc Natl Acad Sci USA 2003;101: 781-786.

21 Hartmann C, Mueller W, Deimling AV. Pathology and molecular genetics of oligodendroglial tumors. J Mol Med 2004;82:638-655.

22 Bao SD, Wu QL, Sathornsumetee S, et al. Stem cell-like glioma cells promote tumor angiogenesis through vascular endothelial growth factor. Cancer Res 2006; 66:7843-7848.

23 Bao SD, Wu QL, McLendon RE, et al. Glioma stem cells promote radioresistance by preferential activation of the DNA damage response. Nature 2006;444:756-760.

24 Porta CL, Alessandri G, Marras C, et al. Glioblastomaderived tumorospheres identify a population of tumor stem-like cells with angiogenic potential and enhanced multidrug resistance phenotype. Glia 2006;54: 850-860.

25 Oliver TG, Wechsler-Reya J. Getting at the root and stem of brain tumors. Neuron 2004;42:885-888.

26 Sanai N, Alvarez-Buylla A, Berger MS. Neural stem cells and the origin of gliomas. $N$ Engl J Med 2005;353:811-822.

27 Wicha MS, Liu SL, Dontu G. Cancer stem cells: an old idea-a paradigm shift. Cancer Res 2006;66: 1883-1890.

28 Al-Hajj M, Clarke MF. Self-renewal and solid tumor stem cells. Oncogene 2004;23:7274-7282.

29 Al-Hajj M, Becker MW, Wicha M, et al. Therapeutic implications of cancer stem cells. Curr Opin Genet Dev 2004;14:43-47. 